EUROPEAN JOURNAL OF PURE AND APPLIED MATHEMATICS

Vol. 12, No. 2, 2019, 270-278

ISSN 1307-5543 - www.ejpam.com

Published by New York Business Global

\title{
Some characterizations of $\beta$-paracompactness in ideal topological space
}

\author{
E. D. Yıldırım¹ ${ }^{1}$ O. B. Özbakır ${ }^{2 *}$ and A.C̣. Güler² \\ ${ }^{1}$ Department of Mathematics, Faculty of Science and Letters, Yaşar University, \\ İzmir, Turkey \\ ${ }^{2}$ Department of Mathematics, Faculty of Science, Ege University, İzmir, Turkey
}

\begin{abstract}
In this paper, we introduce $\beta$-paracompactness with respect to an ideal ( $I$ - $\beta$-paracompactness) as a weak form of $\beta$-paracompactness and $I$-paracompactness. We give some relations between this concept and some other types of paracompactness, and also we study some of its fundamental properties.
\end{abstract}

2010 Mathematics Subject Classifications: 54D20, 54A05, 54C10, 54G05

Key Words and Phrases: $\beta$-paracompact, ideal, $I$ - $\beta$-paracompact, $\sigma$ - $\beta$-locally finite

\section{Introduction}

Paracompactness is one of the important concepts of general topology. In literature, different kinds of generalized paracompactness such as S-paracompactness [5], $P_{3^{-}}$ paracompactness [6] and $\beta$-paracompactness [11] are studied.

The concept of $I$-paracompactness as generalization of paracompactness was given by Zahid [24]. Furthermore, this concept was studied by Hamlet et al. [13] and Sathiyasundari and Renukadevi [22]. Recently, S-paracompactness with respect to an ideal which is weaker form of I-paracompactness was studied by J. Sanabria et al. [21].

Here, we introduce $I$ - $\beta$-paracompactness and we compare this concept with the other types of paracompactness. Then, we give counterexamples showing that the opposite directions of Proposition 1 and 2 do not hold. Furthermore, adding some conditions, we find that the reverse directions may happen to be true. Besides, we investigate some of its essential properties. Finally, we examine $I$ - $\beta$-paracompactness under some functions.

*Corresponding author.

DOI: https://doi.org/10.29020/nybg.ejpam.v12i2.3394

Email addresses: esra.dalan@yasar.edu.tr (E. D. Yıldırım),

oya.ozbakir@ege.edu.tr (O. B. Özbakır), aysegul.caksu.guler@ege.edu.tr (A.C̣. Güler) 


\section{Preliminaries}

Throughout this work, $(X, \tau)$ denotes a topological space on which no separation axioms are assumed unless clearly indicated. If $A$ is a subset of $(X, \tau)$, then the closure of $A$ and the interior of $A$ will be denoted by $\operatorname{cl}(A)$ and $\operatorname{int}(A)$, respectively. Also, the class of all subsets of $X$ will be denoted by $\mathcal{P}(X)$. A subset $A$ of $(X, \tau)$ is said to be semi-open [16] if there exists $U \in \tau$ such that $U \subseteq A \subseteq \operatorname{cl}(U)$. This is equivalent to say that $A \subseteq \operatorname{cl}(\operatorname{int}(A))$. Also, $A$ is said to be $\beta$-open [1] (preopen [18]) if $A \subseteq \operatorname{cl}(\operatorname{int}(\operatorname{cl}(A)))(A \subseteq \operatorname{int}(\operatorname{cl}(A)))$. The concept of $\beta$-open sets is equal to that of semi-preopen sets in [7]. The family of all semiopen (resp. $\beta$-open and preopen) sets of $(X, \tau)$ is denoted by $S O(X, \tau)$ (resp. $\beta O(X, \tau)$ and $P O(X, \tau))$. The complement of a semi-open (resp. $\beta$-open and preopen) set is said to be semi-closed [10] (resp. $\beta$-closed [1, 7] and preclosed [18]). The semi-closure [10] (resp. $\beta$-closure [3, 7] and preclosure[18]) of $A$, denoted by $\operatorname{scl}(A)(\operatorname{resp} . \beta c l(A)$ and $p c l(A))$, is the intersection of all semi-closed (resp. $\beta$-closed and preclosed) sets containing $A$. Note that, $\beta c l(A)$ is $\beta$-closed $[3,7]$.

Lemma 1. [3, 7] For a subset $A$ of a topological space $(X, \tau)$, the following conditions hold:

(i) $x \in \beta c l(A)$ if and only if $A \cap U \neq \emptyset$ for every $U \in \beta O(X, \tau)$ containing $x$,

(ii) $A$ is $\beta$-closed if and only if $A=\beta \operatorname{cl}(A)$.

Theorem 1. [19] Let $(X, \tau)$ be a space, $A \subseteq Y \subseteq X$ and $Y$ be $\beta$-open in $(X, \tau)$. Then $A$ is $\beta$-open in $(X, \tau)$ if and only if $A$ is $\beta$-open in the subspace $\left(Y, \tau_{Y}\right)$.

A function $f:(X, \tau) \rightarrow(Y, \sigma)$ is said to be pre $\beta$-closed [17] (pre $\beta$-open [17]) if for every $\beta$-closed $(\beta$-open)set $A$ of $(X, \tau), f(A)$ is $\beta$-closed $(\beta$-open) in $(Y, \sigma)$ and $f:(X, \tau) \rightarrow(Y, \sigma)$ is said to be $\beta$-irresolute [17] if for every $\beta$-open set $B$ of $(Y, \sigma), f^{-1}(B)$ is $\beta$-open in $(X, \tau)$. If $f:(X, \tau) \rightarrow(Y, \sigma)$ is continuous and open, then $f$ is $\beta$-irresolute and pre $\beta$-open.

Lemma 2. [11] Let $f:(X, \tau) \rightarrow(Y, \sigma)$ be a surjective function. Then $f$ is pre $\beta$-closed if and only if for every $y \in Y$ and every $\beta$-open set $U$ in $(X, \tau)$ which contains $f^{-1}(y)$, there exists a $V \in \beta O(Y, \sigma)$ such that $y \in V$ and $f^{-1}(V) \subseteq U$.

A space $(X, \tau)$ is called extremally disconnected[23](briefly, e. d.) if the closure of every open set in $X$ is open and called submaximal [8] if each dense subset of $X$ is open in $X$.

Lemma 3. [20] $(X, \tau)$ is submaximal if and only if every pre-open set is open.

Lemma 4. [9] $(X, \tau)$ is e.d. if and only if every $\beta$-open set is pre-open.

A collection $\mathcal{V}$ of subsets of a space $(X, \tau)$ is said to be locally finite [23](resp. s-locally finite [4], $\beta$-locally finite [11] and $p$-locally finite[6]), if for each $x \in X$ there exists $U_{x} \in \tau$ (resp. $U_{x} \in S O(X, \tau), U_{x} \in \beta O(X, \tau)$ and $U_{x} \in P O(X, \tau)$ ) containing $x$ and $U_{x}$ intersects at most finitely many members of $\mathcal{V}$. Every locally finite collection of subsets of a space 
$(X, \tau)$ is $\beta$-locally finite[11] and p-locally finite[6]. Also, a collection $\mathcal{A}$ of subsets of a space $(X, \tau)$ is said to be $\sigma$-locally finite if $\mathcal{A}=\bigcup_{n=1}^{\infty} \mathcal{A}_{n}$ where each $\mathcal{A}_{n}$ is locally finite family [13].

Theorem 2. [11] Let $(X, \tau)$ be an e.d. submaximal space. Then every $\beta$-locally finite collection of subsets of $X$ is locally finite.

A space $(X, \tau)$ is said to be $\beta$-compact [2] if every cover of $X$ by $\beta$-open sets has a finite subcover. Also a space $(X, \tau)$ is said to be paracompact [23] (resp. S-paracompact [5], $\beta$-paracompact [11] and $P_{3}$-paracompact [6]), if every open cover of $X$ has a locally finite open (resp. locally finite semi-open, $\beta$-locally finite $\beta$-open and $p$-locally finite preopen) refinement which covers to $X$.

An ideal is defined as a nonempty collection $I$ of subsets of $X$ satisfying the following two conditions:

(1) If $A \in I$ and $B \subseteq A$, then $B \in I$,

(2) If $A \in I$ and $B \in I$, then $A \cup B \in I$.

Given a topological space $(X, \tau)$ with an ideal $I$ on $X$ and if $\mathcal{P}(X)$ is the set of all subsets of $X$, a set operator $(.)^{*}: \mathcal{P}(X) \rightarrow \mathcal{P}(X)$, called a local function [15] of $A$ with respect to $\tau$ and $I$ is defined as follows: for $A \subseteq X, A^{*}(I, \tau)=\{x \in X: V \cap A \notin I$ for every $V \in \tau(x)\}$ where $\tau(x)=\{V \in \tau: x \in V\}$. A Kuratowski closure operator $c l^{*}($.$) for$ a topology $\tau^{*}(I, \tau)$, called the *-topology, finer than $\tau$, is defined by $c l^{*}(A)=A \cup A^{*}(I, \tau)$ [14]. A basis $\beta(I, \tau)$ for $\tau^{*}(I, \tau)$ can be described as follows: $\beta(I, \tau)=\{V-J: V \in \tau$ and $J \in I\}[14]$. We will simply write $A^{*}$ for $A^{*}(I, \tau), \tau^{*}$ or $\tau^{*}(I)$ for $\tau^{*}(I, \tau)$ and $\beta$ for $\beta(I, \tau)$. If $I$ is an ideal on $X$, then $(X, \tau, I)$ is called an ideal topological space.

A space $(X, \tau, I)$ is said to be $I$-paracompact [24] (I-S-paracompact [21]) if every open cover $\mathcal{U}$ of $X$ has a locally finite open (semi-open) refinement $\mathcal{V}$, not necessarily a cover, such that $X-\bigcup\{V: V \in \mathcal{V}\} \in I$. A collection $\mathcal{V}$ of subsets of $X$ such that $X-\bigcup\{V: V \in \mathcal{V}\} \in I$ is called an $I$-cover [24] of $X$. A space $(X, \tau, I)$ is said to be $I$-regular[12] if for each closed set $F$ and a point $p \notin F$, there exist disjoint open sets $U$ and $V$ such that $p \in U$ and $F-V \in I$.

\section{I- $\beta$-paracompactness}

Definition 1. A space $(X, \tau, I)$ is said to be $I$ - $\beta$-paracompact or $\beta$-paracompact with respect to $I$ if every open cover $\mathcal{U}$ of $X$ has a $\beta$-locally finite $\beta$-open refinement $\mathcal{V}$ (not necessarily a cover) such that $X-\bigcup\{V: V \in \mathcal{V}\} \in I$.

$A$ subset $A$ of a space $(X, \tau, I)$ is called an $I$ - $\beta$-paracompact set in $(X, \tau, I)$ if every open cover $\mathcal{U}$ of $A$ has a $\beta$-locally finite (with respect to $\tau$ ) $\beta$-open refinement $\mathcal{V}$ such that $A-\bigcup\{V: V \in \mathcal{V}\} \in I$.

Proposition 1. If $(X, \tau)$ is $\beta$-paracompact, then $(X, \tau, I)$ is I- $\beta$-paracompact.

Proof. It is obvious since $\emptyset \in I$. 
Obviously, every compact space is $I$ - $\beta$-paracompact since every compact space is $\beta$ paracompact [11].

The following example shows that the converse of Proposition 1 may not be true, in general.

Example 1. Let $X=\mathbb{N}$ be the set of natural numbers with the topology $\tau=\{G \subseteq \mathbb{N}: 5 \in$ $G\} \cup\{\emptyset\}$ and the ideal $I=\{U \subseteq \mathbb{N}: 5 \notin U\}$. Observe that $(X, \tau, I)$ is I- $\beta$-paracompact space but $(X, \tau)$ is not $\beta$-paracompact since the collection $\{\{5, x\}: x \in \mathbb{N}\}$ is an open cover of $X$ which admits no $\beta$-locally finite $\beta$-open refinement in $X$.

\section{Remark 1.}

(1) If $I=\{\emptyset\}$, then $(X, \tau, I)$ is $I$ - $\beta$-paracompact if and only if $(X, \tau)$ is $\beta$-paracompact.

(2) If $I=\{\emptyset\}$ and $(X, \tau, I)$ is an e.d. space, then $(X, \tau, I)$ is $I$ - $\beta$-paracompact if and only if $(X, \tau)$ is $P_{3}$-paracompact.

Proposition 2. If $(X, \tau, I)$ is $I$-S-paracompact then it is I- $\beta$-paracompact.

Proof. Since every locally finite collection of subsets of $X$ is $\beta$-locally finite and every semi-open set is $\beta$-open, it is clear.

Clearly, every S-paracompact space is $I$ - $\beta$-paracompact since every S-paracompact space is $I$-S-paracompact[21]. Also, every $I$-paracompact space is $I$ - $\beta$-paracompact since every $I$-paracompact space is $I$-S-paracompact[21].

The following example shows that the converse of Proposition 2 may not be true, in general.

Example 2. Let $X=[0,2] \cup[3,10]$ with the topology $\tau=\{U \subseteq X:[0,2] \subseteq U\} \cup\{\emptyset\}$ and the ideal $I=\{A: A \subseteq[0,2]\}$. Then $(X, \tau, I)$ is $I$ - $\beta$-paracompact since every open cover of $X$ has $\beta$-locally finite $\beta$-open refinement $\mathcal{V}=\{\{x\}: x \in[0,2]\} \cup\{\{y, z\}: y \in[0,2], z \in[3,10]\}$ such that $X-\bigcup\{V: V \in \mathcal{V}\} \in I$. But it is not I-S-paracompact since $\tau=S O(X)$.

Theorem 3. If $(X, \tau, I)$ is an e.d. submaximal I- $\beta$-paracompact space, then it is $I$-Sparacompact.

Proof. It is obvious from Lemma 3, Lemma 4 and Theorem 2.

Theorem 4. If $(X, \tau, I)$ is $I$ - $\beta$-paracompact and $J$ is an ideal on $X$ with $I \subseteq J$, then $(X, \tau, J)$ is $J$ - $\beta$-paracompact.

Proof. Let $(X, \tau, I)$ be $I$ - $\beta$-paracompact and $I \subseteq J . A n d$ let $\mathcal{U}=\left\{U_{\lambda}: \lambda \in \Lambda\right\}$ be an open cover of $X$. Since $(X, \tau, I)$ is $I$ - $\beta$-paracompact, $\mathcal{U}$ has a $\beta$-locally finite $\beta$-open refinement $\mathcal{V}$ such that $X-\bigcup\{V: V \in \mathcal{V}\} \in I$. Since $I \subseteq J, X-\bigcup\{V: V \in \mathcal{V}\} \in J$. Thus, $(X, \tau, J)$ is $J$ - $\beta$-paracompact.

Lemma 5. [11]Let $\mathcal{V}=\left\{V_{\lambda}: \lambda \in \Lambda\right\}$ be a collection of subsets of a space $(X, \tau)$. $\mathcal{V}$ is $\beta$-locally finite if and only if $\left\{\beta c l\left(V_{\lambda}\right): \lambda \in \Lambda\right\}$ is $\beta$-locally finite. 
Lemma 6. If a cover $\mathcal{U}=\left\{U_{\lambda}: \lambda \in \Lambda\right\}$ of a space $(X, \tau, I)$ has a $\beta$-locally finite $\beta$-open refinement $\mathcal{V}$ such that $X-\bigcup\{V: V \in \mathcal{V}\} \in I$ then there exists a $\beta$-locally finite precise $\beta$ - open refinement $\mathcal{H}=\left\{H_{\lambda}: \lambda \in \Lambda\right\}$ of $\mathcal{U}$ such that $X-\bigcup\left\{H_{\lambda}: H_{\lambda} \in \mathcal{H}\right\} \in I$.

Proof. The proof is similar to that of Lemma 1.3 in [21].

Definition 2. A collection $\mathcal{A}$ of subsets of a space $(X, \tau)$ is said to be $\sigma$ - $\beta$-locally finite if $\mathcal{A}=\bigcup_{n=1}^{\infty} \mathcal{A}_{n}$ where each collection $\mathcal{A}_{n}$ is a $\beta$ - locally finite family.

Lemma 7. Every $\beta$-locally finite collection of subsets of a space $(X, \tau)$ is $\sigma$ - $\beta$-locally finite. Proof. It is obvious.

Theorem 5. Let $(X, \tau)$ be a regular space. If $(X, \tau, I)$ is $I$ - $\beta$-paracompact, then every open cover of $X$ has a $\beta$-closed $\beta$-locally finite I-cover refinement.

Proof. Let $\mathcal{U}$ be an open cover of $X$. By regularity of $X$, for each $x \in X$ and $U_{x} \in \mathcal{U}$ containing $x$, there exists an open set $G_{x}$ of $x$ such that $c l\left(G_{x}\right) \subseteq U_{x}$. Then $\mathcal{U}_{1}=\left\{G_{x}: x \in X\right\}$ is an open cover of $X$. Since $X$ is $I$ - $\beta$-paracompact, $\mathcal{U}_{1}$ has $\beta$ locally finite $\beta$-open refinement $\mathcal{V}_{1}=\left\{V_{\lambda}: \lambda \in \Lambda\right\}$ such that $X-\bigcup\left\{V_{\lambda}: \lambda \in \Lambda\right\} \in I$. Then $X-\bigcup\left\{\beta c l\left(V_{\lambda}\right): \lambda \in \Lambda\right\} \in I$. By Lemma $5, \mathcal{V}=\left\{\beta c l\left(V_{\lambda}\right): V_{\lambda} \in \mathcal{V}_{1}\right\}$ is $\beta$ locally finite. Since $\mathcal{V}_{1}$ refines $\mathcal{U}_{1}$, for every $\lambda \in \Lambda$, there is some $G_{x} \in \mathcal{U}_{1}$ such that $V_{\lambda} \subseteq G_{x}$. Then $\beta c l\left(V_{\lambda}\right) \subseteq \operatorname{cl}\left(V_{\lambda}\right) \subseteq \operatorname{cl}\left(G_{x}\right)$ implies $\beta c l\left(V_{\lambda}\right) \subset U_{x}$. Hence $\mathcal{V}$ refines $\mathcal{U}$. So, $\mathcal{V}=\left\{\beta c l\left(V_{\lambda}\right): V_{\lambda} \in \mathcal{V}_{1}\right\}$ is $\beta$-closed $\beta$-locally finite I-cover refinement.

Remark 2. If $(X, \tau, I)$ is considered to be e.d. submaximal regular space, then the Theorem 5 becomes the Theorem 2.20 in [22].

Theorem 6. If $(X, \tau, I)$ is I- $\beta$-paracompact, then every open cover of $X$ has a $\beta$-open $\sigma$ - $\beta$-locally finite I-cover refinement.

Proof. It is obvious by Lemma 7.

Theorem 7. Let $(X, \tau, I)$ be a regular space and $\beta O(X, \tau)$ be closed under finite intersection. Then, $(X, \tau, I)$ is $I$ - $\beta$-paracompact if and only if every open cover of $X$ has a $\beta$-open $\sigma$ - $\beta$-locally finite I-cover refinement.

Proof. To show sufficiency, let $\mathcal{U}$ be an open cover of $\mathrm{X}$. By hypothesis, there exists a $\sigma$ - $\beta$-locally finite $\beta$-open refinement $\mathcal{V}$ of $\mathcal{U}$ such that $X-\bigcup\{V: V \in \mathcal{V}\} \in I$. Also, $\mathcal{V}=\bigcup_{n=1}^{\infty} \mathcal{V}_{n}$ where each collection $\mathcal{V}_{n}$ is a $\beta$ - locally finite. For each $n \in \mathbb{N}$, let $H_{n}$ $=\bigcup\left\{V: V \in \mathcal{V}_{n}\right\}$ so that $X-\bigcup\left\{H_{n}: n \in \mathbb{N}\right\} \in I$. For each $n \in \mathbb{N}$, let $G_{n}=H_{n}-\bigcup_{i=1}^{n-1} H_{i}$. Then $\left\{G_{n}: n \in \mathbb{N}\right\}$ refines $\left\{H_{n}: n \in \mathbb{N}\right\}$. Let $x \in X$, and let $n$ be the smallest member of $\left\{n \in \mathbb{N}: x \in H_{n}\right\}$. Then $x \in G_{n}$ and $X-\bigcup\left\{G_{n}: n \in \mathbb{N}\right\} \in I$. Also, $G_{n x}$ is a $\beta$-open set containing $x$ that intersects only finite family number of members of $G_{n}$ so 
that $\left\{G_{n}: n \in \mathbb{N}\right\}$ is $\beta$-locally finite. Let $\mathcal{O}=\left\{V \cap G_{n}: V \in \mathcal{V}_{n}\right.$ and $\left.n \in \mathbb{N}\right\}$. Since $\left\{G_{n}: n \in \mathbb{N}\right\}$ is $\beta$-locally finite, $\mathcal{O}$ is $\beta$-locally finite. Also, since $\beta O(X, \tau)$ is closed under finite intersection and $\mathcal{V}$ is $\beta$-open refinement of $\mathcal{U}, \mathcal{O}$ is $\beta$-open refinement of $\mathcal{U}$. Then, $X-\bigcup\left\{V \cap G_{n}: n \in \mathbb{N}\right\} \in I$ because $X-\bigcup\left\{G_{n}: n \in \mathbb{N}\right\} \in I$. Thus, $(X, \tau, I)$ is $I$ - $\beta$-paracompact.

Remark 3. If $(X, \tau, I)$ is considered to be e.d. submaximal regular space, then Theorem 7 becomes Theorem 2.22 in [22].

Theorem 8. For any ideal topological space $(X, \tau, I)$, the following are equivalent:

(i) For every closed subset $A$ of $X$ and every $x \notin A$, there exist disjoint $\beta$-open sets $U$ and $V$ such that $x \in U$ and $A-V \in I$.

(ii) For every open subset $G$ of $X$ and every $x \in G$, there exists a $\beta$-open set $U$ such that $x \in U$ and $\beta c l(U)-G \in I$.

Proof. (i) $\Rightarrow$ (ii) Let $G \subseteq X$ be open and $x \in G$. Then $X-G=A$ is closed and $x \notin A$. From (i), there exist disjoint $\beta$-open sets $U$ and $V$ such that $x \in U$ and $A-V \in I$. Since $U$ and $V$ are disjoint, we have $\beta c l(U) \subseteq X-V$. Thus, $A \cap \beta c l(U) \subseteq A-V$. Then, $\beta c l(U) \cap(X-G) \in I$. Therefore, $\beta c l(U)-G \in I$.

(ii) $\Rightarrow$ (i) Let $A \subseteq X$ be closed and $x \notin A$. Then, $X-A=G$ is open and $x \in G$. From (ii), there exists a $\beta$-open set $U$ such that $x \in U$ and $\beta c l(U)-G \in I$. Thus, $X-\beta c l(U)=V \in \beta O(X)$ and $U \cap V=\emptyset$. Furthermore, $A-V=(X-G)-(X-\beta c l(U))=$ $\beta c l(U)-G \in I$.

The following example reveals that for a locally finite collection of subsets of $\mathcal{V}=\left\{V_{\lambda}\right.$ : $\lambda \in \Lambda\}$ of a space $(X, \tau)$, the equality $\operatorname{cl}\left(\bigcup\left\{V_{\lambda}: \lambda \in \Lambda\right\}\right)=\bigcup\left\{\operatorname{cl}\left(V_{\lambda}\right): \lambda \in \Lambda\right\}$ always holds whereas for $\beta$-locally finite collection of subsets $\mathcal{U}=\left\{U_{\lambda}: \lambda \in \Lambda\right\}$ of a space $(X, \tau)$, the equality $\beta c l\left(\bigcup\left\{U_{\lambda}: \lambda \in \Lambda\right\}\right)=\bigcup\left\{\beta c l\left(U_{\lambda}\right): \lambda \in \Lambda\right\}$ does not hold in general.

Example 3. Consider the real number $\mathbb{R}$ with usual topology $\tau$. Let $\mathcal{V}=\{[0,1),(1,2]\}$. Then $\mathcal{V}$ is $\beta$-locally finite in $(\mathbb{R}, \tau)$ since it is finite. But $\beta c l([0,1) \cup(1,2]) \neq \beta \operatorname{cl}([0,1)) \cup$ $\beta c l((1,2])$.

Theorem 9. Suppose that for a $\beta$-locally finite collection of subsets $\mathcal{V}=\left\{V_{\lambda}: \lambda \in \Lambda\right\}$ of a space $(X, \tau, I)$, the equality $\beta c l\left(\bigcup\left\{V_{\lambda}: \lambda \in \Lambda\right\}\right)=\bigcup\left\{\beta c l\left(V_{\lambda}\right): \lambda \in \Lambda\right\}$ holds. If $(X, \tau, I)$ is Hausdorff $I$ - $\beta$-paracompact, then for every closed subset $A$ of $X$ and every $x \notin A$, there exist disjoint $\beta$-open sets $U$ and $V$ such that $x \in U$ and $A-V \in I$.

Proof. Let $A \subseteq X$ closed and $x \notin A$. Since $X$ is Hausdorff space, there exists an open set $H_{y}$ containing y for each $y \in A$ such that $x \notin \operatorname{cl}\left(H_{y}\right)$. Thus, $\mathcal{H}=\left\{H_{y}: y \in\right.$ $A\} \cup\{X-A\}$ is an open cover of $X$. By hypothesis and Lemma 6, $\mathcal{H}$ has a $\beta$-locally finite precise $\beta$-open refinement $\mathcal{W}=\left\{W_{y}: y \in A\right\} \cup\{G\}$ such that $W_{y} \subseteq H_{y}$ for each $y \in A$, $G \subseteq X-A$ and $X-\left(\bigcup\left\{W_{y}: y \in A\right\} \cup\{G\}\right) \in I$. Since $A-\left(\bigcup\left\{W_{y}: y \in A\right\}\right)=A-\left(\bigcup\left\{W_{y}:\right.\right.$ $y \in A\} \cup\{G\}) \subseteq X-\left(\bigcup\left\{W_{y}: y \in A\right\} \cup\{G\}\right)$, we have $A-\left(\bigcup\left\{W_{y}: y \in A\right\}\right) \in I$. Let 
we say $V=\bigcup\left\{W_{y}: y \in A\right\}$. Then, $V$ is $\beta$-open set in $X$ and $A-V \in I$. Since $x \notin \operatorname{cl}\left(H_{y}\right)$, we have $x \notin \operatorname{cl}\left(W_{y}\right)$. This implies that $x \notin \beta c l\left(W_{y}\right)$. Since $\mathcal{W}$ is $\beta$-locally finite, $\beta c l(V)=\beta c l\left(\bigcup\left\{W_{y}: y \in A\right\}\right)=\bigcup\left\{\beta c l\left(W_{y}\right): y \in A\right\}$ by hypothesis. Thus, for a $\beta$-open set $U=X-\beta c l(V)$, we have $U \cap V=\emptyset$ such that $x \in U$.

From Theorem 8 and Theorem 9, we have the following Corollary.

Corollary 1. If $(X, \tau, I)$ is an e.d. submaximal Hausdorff $I$ - $\beta$-paracompact space, then $(X, \tau, I)$ is I-regular.

Theorem 10. Let $A$ and $B$ be subsets in ideal topological space $(X, \tau, I)$. If $A$ is $I-\beta$ paracompact set in $X$ and $B$ is closed in $X$, then $A \cap B$ is $I$ - $\beta$-paracompact set in $X$.

Proof. Let $\mathcal{U}=\left\{U_{\lambda}: \lambda \in \Lambda\right\}$ be an open cover of $A \cap B$. Since $X-B$ is open in $X, \mathcal{U}^{\prime}=\left\{U_{\lambda}: \lambda \in \Lambda\right\} \cup\{X-B\}$ is open cover of $A$. By hypothesis and Lemma $6, \mathcal{U}^{\prime}$ has a $\beta$ - locally finite precise $\beta$-open refinement $\left\{V_{\lambda}: \lambda \in \Lambda\right\} \cup\{V\}$ such that $V_{\lambda} \subseteq U_{\lambda}$ for each $\lambda \in \Lambda, V \subseteq X-B$ and $A-\left(\bigcup\left\{V_{\lambda}: \lambda \in \Lambda\right\} \cup\{V\}\right) \in I$. Since $(A \cap B)-\left(\bigcup\left\{V_{\lambda}: \lambda \in \Lambda\right\}\right)=(A \cap B)-\left(\bigcup\left\{V_{\lambda}: \lambda \in \Lambda\right\} \cup\{V\}\right) \subseteq A-\left(\bigcup\left\{V_{\lambda}: \lambda \in \Lambda\right\} \cup\{V\}\right)$, we have $(A \cap B)-\left(\bigcup\left\{V_{\lambda}: \lambda \in \Lambda\right\}\right) \in I$. Hence, $A \cap B$ is I- $\beta$-paracompact set in $X$.

Corollary 2. Let $(X, \tau, I)$ be an $I$ - $\beta$-paracompact space and $A \subseteq X$. If $A$ is closed in $X$, then $A$ is an $I-\beta$-paracompact set in $X$.

Lemma 8. [13] If $I \neq \emptyset$ is an ideal on $X$ and $Y$ is a subset of $X$, then $I_{Y}=\{Y \cap G \mid G \in$ $I\}=\{G \in I \mid G \subseteq Y\}$ is an ideal on $Y$.

Theorem 11. Let $A$ and $B$ be subsets in ideal topological space $(X, \tau, I)$ such that $B \subseteq A$. If $A$ is $\beta$-open in $X$ and $B$ is an $I_{A}$ - $\beta$-paracompact set in $A$ then $B$ is an $I$ - $\beta$-paracompact set in $X$.

Proof. Let $\mathcal{U}=\left\{U_{\lambda}: \lambda \in \Lambda\right\}$ be an open cover of $B$ in $X$. Then, $\mathcal{U}_{\mathcal{B}}=\left\{U_{\lambda} \cap A: \lambda \in \Lambda\right\}$ is an open cover of $B$ in $A$. Since $B$ is an $I_{A}-\beta$-paracompact set in $A, \mathcal{U}_{\mathcal{B}}$ has a $\beta$-locally finite precise $\beta$-open refinement $\mathcal{V}_{\mathcal{B}}$ in $A$ such that $B-\bigcup\left\{V_{\lambda}: V_{\lambda} \in \mathcal{V}_{\mathcal{B}}\right\} \in I_{A}$. Thus, $\mathcal{V}_{\mathcal{B}}$ is a $\beta$-locally finite precise $\beta$-open refinement in $X$ by Theorem 1. Also, $B-\bigcup\left\{V_{\lambda}: V_{\lambda} \in\right.$ $\left.\mathcal{V}_{\mathcal{B}}\right\} \in I$. Hence, $B$ is an $I$ - $\beta$-paracompact set in $X$.

Theorem 12. Let $f:(X, \tau, I) \rightarrow(Y, \sigma, J)$ be a continuous, open and pre $\beta$-closed surjection with $f^{-1}(y) \beta$-compact for every $y \in Y$ and $f(I) \subseteq J$. If $(X, \tau, I)$ is $I$ - $\beta$-paracompact, then $(Y, \sigma, J)$ is $J$ - $\beta$-paracompact.

Proof. Let $\mathcal{U}=\left\{U_{\lambda}: \lambda \in \Lambda\right\}$ be an open cover of $Y$. Then, $\left\{f^{-1}\left(U_{\lambda}\right): \lambda \in \Lambda\right\}$ is an open cover of $X$. Since $(X, \tau, I)$ is $I$ - $\beta$-paracompact, this open cover has a $\beta$-locally finite precise $\beta$-open refinement $\mathcal{V}=\left\{V_{\lambda}: \lambda \in \Lambda\right\}$ such that $X-\bigcup\left\{V_{\lambda}: V_{\lambda} \in \mathcal{V}\right\} \in I$. Since $f$ is pre $\beta$-open, $f(\mathcal{V})=\left\{f\left(V_{\lambda}\right): \lambda \in \lambda\right\}$ is a precise $\beta$-open refinement of $\mathcal{U}$. Also, $Y-\bigcup\left\{f\left(V_{\lambda}\right): \lambda \in \Lambda\right\} \in J$. Now, let we prove that $f(\mathcal{V})$ is $\beta$-locally finite. Let $y \in Y$. Since $\mathcal{V}$ is $\beta$-locally finite, for $x \in f^{-1}(y)$, there exists a $\beta$-open set $G_{x}$ containing $x$ such that $G_{x}$ intersects at most finitely members of $\mathcal{V}$. Since $f^{-1}(y)$ is $\beta$-compact, $\left\{G_{x}: x \in\right.$ 
$\left.f^{-1}(y)\right\}$ has a finite subcollection $H_{y}$ such that $f^{-1}(y) \subseteq \bigcup H_{y}$ and $\bigcup H_{y}$ intersects at most finitely members of $\mathcal{V}$. By Lemma 2 , there exists a $\beta$-open set $W_{y}$ containing y such that $f^{-1}\left(W_{y}\right) \subseteq \bigcup H_{y}$. Then, $f^{-1}\left(W_{y}\right)$ intersects at most finitely members of $\mathcal{V}$. This implies that $W_{y}$ intersects at most finitely members of $f(\mathcal{V})$. Hence, $f(\mathcal{V})$ is $\beta$-locally finite in $Y$. So, $(Y, \sigma, J)$ is $J$ - $\beta$-paracompact.

Theorem 13. Let $f:(X, \tau, I) \rightarrow(Y, \sigma, J)$ be an open, $\beta$-irresolute bijective mapping and $I=f^{-1}(J)$. If $A$ is $J$ - $\beta$-paracompact in $Y$, then $f^{-1}(A)$ is $I$ - $\beta$-paracompact in $X$.

Proof. Let $\mathcal{U}=\left\{U_{\lambda}: \lambda \in \Lambda\right\}$ be an open cover of $f^{-1}(A)$. Since $f$ is open, $\mathcal{U}_{1}=$ $\left\{f\left(U_{\lambda}\right): \lambda \in \Lambda\right\}$ is an open cover of $A$. By hypothesis, this open cover has a $\beta$-locally finite precise $\beta$-open refinement $\mathcal{V}_{1}=\left\{V_{\lambda}: \lambda \in \Lambda\right\}$ such that $A-\bigcup\left\{V_{\lambda}: \lambda \in \Lambda\right\} \in J$. Then, $f^{-1}(A)-\bigcup\left\{f^{-1}\left(V_{\lambda}\right): \lambda \in \Lambda\right\} \in f^{-1}(J)=I$. Since $f$ is $\beta$-irresolute, $\mathcal{V}=\left\{f^{-1}\left(V_{\lambda}\right)\right.$ : $\lambda \in \Lambda$ \} is $\beta$-locally finite $\beta$-open. Let $f^{-1}\left(V_{\lambda}\right) \in \mathcal{V}$. Since $\mathcal{V}_{1}$ refines $\mathcal{U}_{1}$, there exists $f\left(U_{\lambda}\right) \in \mathcal{U}_{1}$ such that $V_{\lambda} \subseteq f\left(U_{\lambda}\right)$. Then $f^{-1}\left(V_{\lambda}\right) \subseteq f^{-1}\left(f\left(U_{\lambda}\right)\right)=U_{\lambda}$. Hence $\mathcal{V}$ refines $\mathcal{U}$. Therefore $f^{-1}(A)$ is $I$ - $\beta$-paracompact in $X$.

\section{Acknowledgements}

The author would like to thank the referees for their helpful suggestions.

\section{References}

[1] Abd El-Monsef, M. E., El-Deeb, S. N. and Mahmoud, R. A. $\beta$-open sets and $\beta$ continuous mapping, Bull. Fac. Sci. Assiut Univ. 12, 77-90, 1983.

[2] Abd El-Monsef, M. E. and Kozae, A. M. Some generalized forms of compactness and closedness, Delta J. Sci. 9, 257-269, 1985.

[3] Abd El-Monsef, M. E., Mahmoud, R. A. and Lashin, E. R. $\beta$-closure and $\beta$-interior, J. Fac. Ed. Ain Shams Univ. 10, 235-245, 1986.

[4] Al-Zoubi, K. Y. s-expandable spaces, Acta Math. Hungar 102(3), 203-212, 2004.

[5] Al-Zoubi, K. Y. S-paracompact spaces, Acta Math. Hungar 110(1-2), 165-174, 2006.

[6] Al-Zoubi, K. and Al-Ghour, S. On P-paracompact spaces, Int. J. Math. Math. Sci. 2007, 1-16, 2007.

[7] Andrijević, D. Semipreopen sets, Mat. Vesnik 38, 24-32, 1986.

[8] Bourbaki, N. General topology, Part I., Addison-Wesley, Reading, Mass. 1966.

[9] Cao, J., Ganster, M. and Reilly, I. Submaximality, extremal disconnectedness and generalized closed sets, Houston Journal of Mathematics 24(4), 681-688, 1998. 
[10] Crossely, S. G. Semi-closed and semi-continuity in topological spaces, Texas J. Sci. 22, 123-126, 1971.

[11] Demir, I. and Ozbakir, O. B. On $\beta$-paracompact spaces, Filomat 27:6, 971-976, 2013.

[12] Hamlet, T. R. and Janković, D. On weaker forms of paracompactness, countable compactness, and Lindelöfness, Ann. New York Acad. Sci., 728, 41- 49, 1994.

[13] Hamlet, T. R., Rose, D. and Janković, D. Paracompactness with respect to an ideal, Internat. J. Math. and Math. Sci. 20(3), 433-442, 1997.

[14] Janković, D. and Hamlett, T. R. New topologies from old via ideals, Amer. Math. Montly 97, 295-310, 1990.

[15] Kuratowski, K. Topologie I, Warszawa 1933.

[16] Levine, N. Semi-open sets and semi-continuity in topological spaces, Amer. Math. Monthly 70, 36-41, 1963.

[17] Mahmoud, R. A. and Abd El-Monsef, M. E. $\beta$-irresolute and $\beta$-topological invariant, Proc. Pakistan Acad. Sci. 27, 285- 296, 1990.

[18] Mashhour, A.S., Abd El-Monsef, M. E. and El-Deeb, S. N. On precontinuous and weak precontinuous mappings, Proc. Math. and Phys. Soc. Egypt 51, 47-53, 1981.

[19] Navalagi, G. B. Semi-precontinuous functions and properties of generalized preclosed sets in topological spaces, Int. J. Math. Math. Sci. 29, 85-98, 2002.

[20] Reilly, I. L. and Vamanamurthy, M. K. On some questions concerning preopen sets, Kyungpook Math. J. 30, 87-93, 1990.

[21] Sanabria, J., Rosas, E., Carpintero, C. , Salas-Brown, M. and García, O. Sparacompactness in ideal topological spaces, Mat. Vesnik 68(3), 192- 203, 2016.

[22] Sathiyasundari, N. and Renukadevi, V. Paracompactness with respect to an ideal, Filomat 27(2), 333-339, 2013.

[23] Willard, S. General topology, Addison-Wesley Publishing Company 1970.

[24] Zahid, M. I. Para H-closed spaces, locally para H-closed spaces and their minimal topologies, Ph. D. Dissertation, Univ. of Pittsburgh 1981. 